\title{
Review Article \\ Current Progress and Challenges in the Search for Autism Biomarkers
}

\author{
Irina Voineagu' ${ }^{1}$ and Hee Jeong Yoo ${ }^{2}$ \\ ${ }^{1}$ School of Biotechnology and Biomolecular Sciences, University of New South Wales, Kensington, Sydney, NSW 2052, Australia \\ ${ }^{2}$ Department of Psychiatry, Seoul National University Bundang Hospital, Gyeonggi 441-701, Republic of Korea
}

Correspondence should be addressed to Hee Jeong Yoo; hjyoo@snu.ac.kr

Received 7 February 2013; Accepted 6 April 2013

Academic Editor: Daniel Martins-de-Souza

Copyright ( 2013 I. Voineagu and H. J. Yoo. This is an open access article distributed under the Creative Commons Attribution License, which permits unrestricted use, distribution, and reproduction in any medium, provided the original work is properly cited.

Autism spectrum disorders (ASD) encompass a range of neurodevelopmental conditions that are clinically and etiologically very heterogeneous. ASD is currently diagnosed entirely on behavioral criteria, but intensive research efforts are focused on identifying biological markers for disease risk and early diagnosis. Here, we discuss recent progress toward identifying biological markers for ASD and highlight specific challenges as well as ethical aspects of translating ASD biomarker research into the clinic.

\section{Introduction}

Autism is a neurodevelopmental disorder diagnosed primarily on clinical criteria. The core clinical manifestations of autism consist of deficits of social communication, language impairments, and repetitive-restrictive behaviors. Comorbid conditions such as intellectual disability, epilepsy, anxiety, and depression are frequently associated with autism [1]. The hallmark of autism's clinical picture is its marked heterogeneity: no two autism patients are alike. Each autistic individual presents with a unique combination of symptom severity in the core domains and a variable mix of comorbid conditions. The clinical heterogeneity of autism, encompassing large variations in disease severity from markedly impaired individuals who need permanent care, to highly functioning patients who fulfill higher education and are entirely self-sufficient, has led to the concept that autism is in fact a spectrum of conditions, rather than a single disease [2]. It is worth noting that mild impairments in language abilities and social communication are also observed as normal variation in the general population and are more frequent among relatives of autistic individuals [2], further supporting the concept that autism encompasses a spectrum of phenotypic variation.

The current Diagnostic and Statistical Manual of Mental Disorders (DSM-IV TR [3]) defines several distinct pervasive developmental disorders: Asperger syndrome, autistic disorder, pervasive developmental disorder not otherwise specified (PDD-NOS), childhood disintegrative disorder (CDD), and Rett syndrome. The first three conditions are generally included under the definition of autism spectrum disorders (ASD). While autistic disorder consists of deficits in all three core domains: language, social interaction, and repetitiverestrictive behaviors, and is often associated with cognitive deficits, Asperger syndrome patients have normal language development and normal cognition. Patients with PDD-NOS have deficits in at least one of the core domains but do not meet the clinical criteria for autism or any of the other pervasive developmental disorders. ASD are four times more prevalent among males than among females, and the overall prevalence of ASD has increased in recent years to a current estimate of $0.5 \%-1 \%$ depending on the study and world area [4].

Recent research has shown that although different clinicians assess the symptoms of a given patient in a very similar manner, the diagnostic ascertainment of these symptoms as autistic disorder, Asperger syndrome, or PDD-NOS varies from clinician to clinician [5]. In addition, the recent increase in ASD prevalence might partially reflect insufficient specificity of current diagnostic criteria [6]. In order to increase the specificity and reduce the variability of ASD diagnosis, the 
updated DSM-V manual [6] proposes significant revisions of autism classification and diagnostic criteria. A detailed discussion of the updated DSM-V criteria is beyond the scope of this review and has been addressed by several recent papers $[6,7]$. In brief, instead of defining three distinct conditions: Asperger syndrome, autistic disorder, and PDD-NOS, DSM$\mathrm{V}$ proposes a single diagnosis of ASD. The DSM-V ASD diagnosis is based on two core domains rather than three: social communication (which includes language and social behavior) and repetitive-restrictive behaviors. ASD is then further subclassified in three levels of severity (levels 1-3). In addition, a novel category of Social Communication Disorder will be added, describing individuals with significant social and communication difficulties similar to those observed in ASD, but without repetitive or restricted behaviors [3].

Several aspects of the updated DSM-V diagnostic criteria are relevant to our discussion of ASD biomarkers, given that the specificity of novel biomarkers can only be as good as the standard diagnostic criteria, used for selecting the research cohorts [8]. First, the need for majorly revised ASD diagnostic criteria highlights the fact that conceptual understanding of autistic symptomatology is still developing in the clinical community. Thus, significant variability in the composition of ASD case cohorts from various studies should be taken into account when interpreting the ASD biomarker data. Second, the inclusion of all three ASD disorders under a single diagnosis might lead to future research cohorts being phenotypically less homogeneous, unless the study design includes careful examination of the symptomatology in all core domains. Asperger patients and patients with deficits restricted to one of the core domains will receive the same diagnosis as patients with full-blown autism and may be included in the same research cohort. On the other hand, the DSM-V criteria are stricter than the previous DSM-IV criteria, and thus, patients diagnosed with ASD by DSM-V may be more homogeneous in terms of severity, regardless of the specific symptomatology that contributes to the disease. A recent study noted that many of the milder Asperger and PDD-NOS cases diagnosed using DSM-IV criteria would not be diagnosed with ASD by DSM-V [7], but this observation remains to be confirmed after DSM-V criteria become widely used.

Identifying biomarkers for ASD has been the focus of intense research since the first description of the disease in the early 1940s [9], but no ASD biological marker has yet demonstrated enough sensitivity and specificity to be translated into the clinic. This review begins with an overview of the specific needs and challenges of identifying biomarkers for ASD and then discusses recent advances toward biomarker development for this complex disorder.

\section{General Considerations on ASD Biomarkers}

Broadly defined, a biomarker is "a characteristic that is objectively measured and evaluated as an indicator of normal biological processes, pathogenic processes, or pharmacologic responses to a therapeutic intervention" [10]. Thus, disease biomarkers include any measurable characteristic, such as DNA sequence variation, MRI imaging, and blood and urine

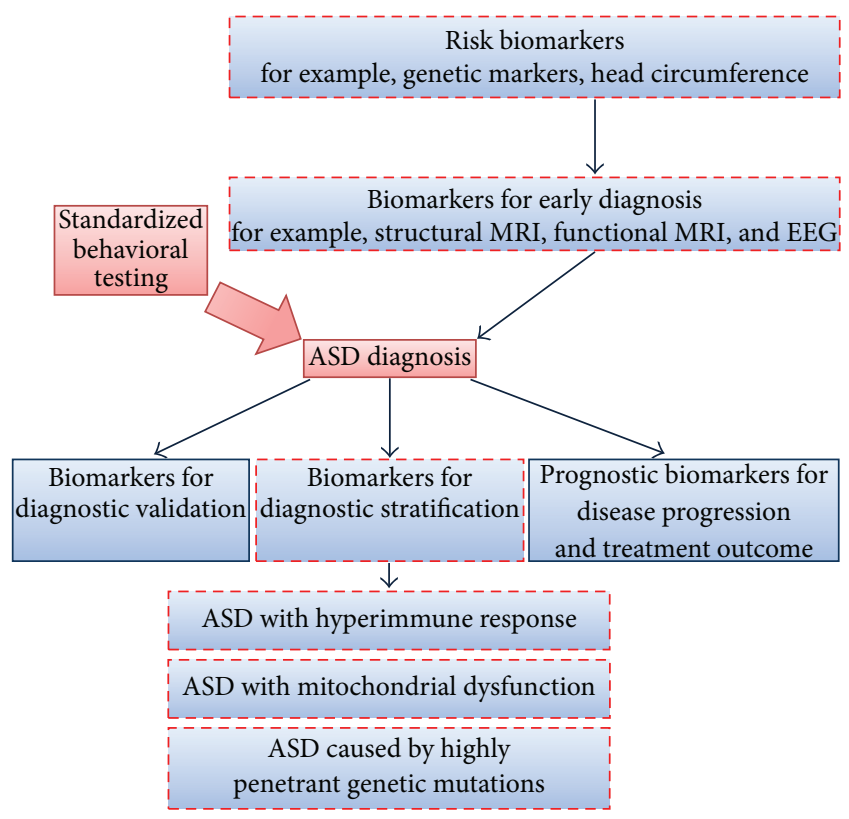

FIGURE 1: Schematic classification of ASD biomarkers and their stage of development. Biomarkers shown with a solid red border (genetic markers for syndromes with high incidence of ASD) are already being used in the clinic; biomarker classes shown with a dashed red border are the subject of intensive research and show preliminary encouraging results; biomarker classes shown with a dark blue border are yet under development. The red arrow highlights the fact that at present the ASD diagnosis is established solely on standardized behavioral criteria. Risk biomarkers and biomarkers for early diagnosis may be applied before standard behavioral testing, while biomarkers for diagnostic validation and stratification and prognostic biomarkers would be employed after establishing an ASD diagnosis based on behavioral testing.

metabolites, can be used as an indicator of disease risk, diagnosis, or prognostic (Figure 1).

(i) Risk biomarkers are used for identifying individuals at risk for developing the disease and often include genetic markers.

(ii) Diagnostic biomarkers are aimed toward early diagnosis (by screening the general population or a selected group in order to diagnose the disease before symptomatology occurs), diagnostic validation, and diagnostic stratification.

(iii) Prognostic biomarkers are biological markers that aid in predicting the disease progression and treatment outcome [11].

Given the lack of specific pharmacological therapy for ASD and the clinical heterogeneity of the disease, the current research efforts are geared mainly toward identification of risk biomarkers and markers for early diagnosis. The most effective therapeutic intervention currently available for ASD is early behavioral therapy [12-14]. While the symptomatology of ASD is usually not apparent until 2 years of age, an additional lag time occurs between the moment when parents become worried about their child and the time the child receives a diagnosis and is enrolled in behavioral therapy [15]. 
Therefore, the short-term goals of ASD biomarker research are (a) to identify risk biomarkers, which can then define a population pool to be screened for early diagnosis, and (b) to develop effective biological and/or behavioral measures that allow diagnosis and early intervention before the full clinical picture develops. Identification of biomarkers for early diagnosis would also aid in eliminating the time lag between referral and diagnosis. In the long term, it would be very valuable to validate ASD diagnosis on an objective biological marker, much like cancer diagnosis is suspected on clinical grounds and validated on biological tests. However, this goal has not yet been achieved for any of the psychiatric disorders, and biological markers are unlikely to replace behavioral testing for standard ASD diagnosis in the next few years. The current research focus is rather on defining strong behavioral diagnostic criteria for ASD, which in turn inform effective biomarker studies.

One of the puzzles faced by ASD research in general, which certainly applies to biomarker research as well, results from the marked disease heterogeneity [16]. As mentioned previously, it has been recognized that there are wide differences in the clinical manifestations of ASD patients, and it is reasonable to assume that the biology underlying the disease in a patient with language and social impairment may be entirely different from the biological mechanisms leading to a clinical picture of intellectual disability, seizures, and repetitive behaviors. Thus, ASD researchers often face the question of whether to investigate a narrow subset of ASD cases, all of whom share similar clinical manifestations, or aim at obtaining more generalizable results by investigating the wider ASD spectrum.

Subphenotyping, that is, selecting a subgroup of ASD patients based on their common clinical manifestations, has proven to be a fruitful research avenue in ASD genetics [17] and may also prove valuable in the search for ASD biomarkers [18] by reducing the cohort sizes necessary for obtaining statistically significant results. It has also been proposed that due to the much higher incidence of ASD in males than in females, the underlying disease mechanisms may be at least to some extent gender specific [19]. Some studies thus subgroup the study cohort by gender in order to increase the likelihood of identifying ASD biomarkers [20]. Conversely, other studies aim to identify biomarkers that can subgroup ASD patients in a manner relevant for prognosis and therapeutics, which is not obvious from the clinical picture. For instance, multiple studies have demonstrated altered immune responses in ASD patients (see below), opening an active area of research on immune biomarkers that can distinguish ASD patients who might benefit from specifically targeted therapy.

The second approach, which aims to identify commonalities among ASD patients is more challenging. Current research from genetic studies as well as research on structural and functional brain MRI indicate that identifying biological changes common to the majority of ASD patients requires large cohort sizes and often a panel of markers, rather than a single marker. As a result, multivariate analyses [21] (such as support vector machine algorithms) are progressively replacing univariate analyses in many areas of ASD biomarker research, from genomics to structural and functional brain imaging. Instead of comparing measurements of a single biological marker between disease and control groups, multivariate analyses are based on the notion that several variables of a certain type (such as the expression level of multiple genes) may be necessary in order to discriminate between disease and normal states. Multivariate analyses thus aim to identify a pattern of data variation in a complex dataset, that best discriminates between disease and control groups. Typically, such a "classifier" pattern is obtained by training an algorithm on data generated from ASD and control individuals, and its ability to discriminate between disease and normal states is then tested on a second, independent set of ASD and controls [21].

Many biological markers for ASD have been proposed to date [22], but with the exception of highly penetrant genetic changes, none have yet advanced to clinic or been consistently reproduced. Conceivably some of the reasons behind the variability of ASD biomarker results are (a) small cohort sizes, (b) small differences between disease and control groups that do not stand replication, (c) lack of replication of results in an independent test group, (d) clinical heterogeneity, and (e) disease variability along developmental trajectory, leading to biological markers being specific for certain age groups or developmental windows. In the following sections, we discuss the main directions taken by research on ASD biomarkers and highlight studies that have attempted to evaluate the sensitivity and specificity of the proposed biological markers.

\section{Brain Imaging Biomarkers}

Studies using structural magnetic resonance imaging (MRI) have attempted to identify differences in brain structure associated with ASD. These studies have been applied to adult ASD cases, aiming to identify diagnostic markers, as well as children with ASD, and infants with a family history of ASD, in a search for markers of early diagnosis. Structural brain changes observed in ASD patients (reviewed in [23]) include increased total brain volume in young ASD children [24], increased frontal lobe volume $[25,26]$, increased cortical thickness in temporal and parietal lobes in ASD children, and decreased cortical thickness in ASD adolescents and adults, as well as structural changes of corpus callosum, basal ganglia, amygdala, and cerebellum [23]. However, none of these changes have been reliably replicated in order to become valuable as ASD biomarkers.

Research focus has lately shifted from comparing individual brain regions between ASD cases and controls to performing multivariate analyses, using structural brain imaging data from multiple brain regions. This approach is supported by the notion that ASD likely affects more than one brain region in any individual and affects the same brain region to a different extent in different ASD patients. Ecker and colleagues [27] have used an SVM approach to analyze whole brain data from 22 adult ASD cases and 22 matched controls in order to identify spatially distributed networks of brain regions with structural properties that could discriminate between ASD cases and controls. This study identified brain networks including limbic, frontal-striatal, frontotemporal, frontoparietal, and cerebellar systems, which could correctly 
classify $86 \%$ of ASD cases using grey matter scans and $68 \%$ of ASD cases using white matter scans. This study used a leave-two-out approach for cross validation. More recently, Uddin et al. [28] used multivariate pattern analysis applied to structural MRI data from children and adolescents with ASD. This study built a classifier based on grey matter in the posterior cingulate cortex, medial prefrontal cortex, and bilateral medial temporal lobes, which reached an accuracy around $90 \%$ in discriminating between the ASD and control groups.

Diffusion tensor imaging (DTI), an MRI method that analyzes white matter microstructure, also demonstrated measurable changes in ASD subjects: decreased fractional anisotropy, reflecting reduced coherence in fiber tract directionality, was observed in several brain regions including ventromedial prefrontal cortex, orbitofrontal cortex, and superior temporal gyrus $[23,29,30]$. A diagnostic classifier built using SVM applied to DTI data demonstrated a prediction accuracy of $80 \%$ [29].

Functional MRI (fMRI) is an imaging method that captures patterns of brain activation and has contributed greatly to the overall understanding of functional brain abnormalities that underlie autism and related disorders (reviewed in [23, 31]). Studies of ASD brain activation during social cognition tasks have demonstrated brain activation changes in the fusiform face area (FFA) in response to face processing [32-34], decreased FFA and amygdala activation during emotional face tasks [35, 36], and impaired activation of the mirror neuron system [37]. Studies of neural correlates of language development in ASD children have shown an abnormal right hemisphere lateralization of temporal cortex activation during language tasks $[23,38]$. fMRI studies of ASD patients also demonstrated decreased long-distance connectivity between brain regions during resting state [39, 40].

Anderson and colleagues used pairwise functional connectivity data from 7266 brain regions across the entire grey matter to build a diagnostic classifier for a set of 40 ASD subjects and 40 matched controls [41]. This classifier had $83 \%$ sensitivity and $75 \%$ specificity ( $79 \%$ accuracy) on the initial dataset and $71 \%$ accuracy in a replication dataset of 21 individuals. Interestingly, the classification accuracy was $89 \%$ and $91 \%$ for the two datasets, respectively, when the classifier was applied only to individuals younger than 20 years, suggesting that functional connectivity differences between ASD and controls diminish after 20 years of age [41].

While these results are very exciting and offer hope for an objective measure that could help ASD diagnosis, the structural and functional imaging biomarkers await replication in larger cohorts and multicenter studies, before translation to clinic becomes feasible.

\section{Electrophysiological Biomarkers}

MRI-based methods and fMRI in particular are expensive and laborious investigations. By comparison, electrophysiological methods are less costly and would be easier to implement in the clinic. Thus, several studies focused on identifying electrophysiological changes associated with ASD [42-45].

Event-related potentials (e.g., electrical brain response to faces) were shown to be delayed in children with ASD, and this measure of brain activity was reposted to be normalized in response to early behavioral interventions $[43,45]$. Delays in auditory evoked responses in superior temporal gyrus were also proposed as a potential biomarker for autism with a sensitivity of $75 \%$ and a specificity of $81 \%$ for discriminating between 25 children with ASD and 17 controls [44].

Recently, Bosl and colleagues [42] proposed that EEG complexity could be used as a biomarker for ASD risk. This study used resting state EEG data from normally developing children and children at high risk for autism, defined as children having an older sibling diagnosed with autism. By calculating a modified multiscale entropy (mMSE) measure and applying an SVM algorithm, the authors were able to discriminate between the high-risk and control groups with $80 \%$ accuracy. It has been objected that this study did not demonstrate that children at high risk for ASD eventually do develop the disease [46]. Moreover, the observed EEG differences might in fact reflect brain adaptive responses to genetic vulnerability. While studies attempting to determine early brain changes in ASD children are very valuable, careful interpretation of these results is necessary given their ethical implications [46].

\section{Genetic Markers}

Initial autism twin studies demonstrated that ASD is highly heritable, with disease concordance rates of $70-90 \%$ among monozygotic twins and 6-10\% among dizygotic twins [47, 48]. More recent estimates show somewhat lower heritability rates than the initial studies (77\% for ASD male monozygotic twins and 50\% for female ASD monozygotic twins [49]) but still support the notion that ASD is highly heritable. Given the high heritability of ASD, intense research efforts have been aimed at uncovering the genetic basis of ASD, and identifying genetic markers that estimate the disease risk. Current research on ASD genetics has been reviewed elsewhere $[2,16,17]$, and here, we focus on the data relevant for potential translation of genetic research into disease markers.

The discovery of several single gene mutations and cytogenetic abnormalities with high ASD prevalence has led to the possibility of identifying a genetic cause of ASD in as many as $20 \%$ of patients [2]. Chromosomal microarray analysis (CMA) using either comparative genomic hybridization (CGH) arrays or single nucleotide polymorphism (SNP) arrays can identify copy number variations (CNVs) such as microdeletions and microduplications in $5-10 \%$ of ASD patients [50]. Thus, it has been proposed that CMA should be used as a first-tier clinical diagnostic test for ASD $[51,52]$. While CMA is not intended for diagnosing ASD, it can be used to investigate the genetic cause of the disease, once the ASD clinical diagnosis has been established. Heil and Schaaf [51] proposed an algorithm for ASD clinical genetic diagnosis, which employs CMA as a screening tool. The interpretation of a positive CMA result (i.e., the identification 
of one or more CNVs in an ASD patient) is complicated by the fact that not all CNVs are pathogenic. Several CNVs have been associated with ASD as being more frequent in the ASD population than in the general population [16]. If a CNV previously associated with ASD is identified by CMA analysis, it should be considered as contributing to the disease and taken into account for genetic counseling for the family [51]. If the CNV identified by CMA has not been previously associated with ASD, it is recommended that the parents of the ASD patient be tested by CMA as well, in order to determine whether the CNV is inherited or de novo. A de novo CNV or a CNV inherited from a parent with a clinical psychiatric disorder is more likely to be causal for ASD. Finally, if a copy number variant (CNV) is not identified by CMA, screening of specific genes for point mutations or other types of genetic changes not detectable by CMA is recommended in patients with a clinical picture suggestive of syndromic forms of ASD.

Syndromic forms of ASD are recognizable clinical syndromes that have high ASD prevalence and often a known genetic cause. Cytogenetic abnormalities such as $\operatorname{dup}(15 \mathrm{q})$, as well as single gene mutations affecting CNTNAP2 (cortical dysplasia focal epilepsy syndrome), CACNA1C (Timothy syndrome), MECP2 (Rett syndrome), and FMR1 (fragile X syndrome), are associated with ASD in more than $50 \%$ of the cases [2]. These highly penetrant genetic changes are valuable markers for subclassifying ASD. Animal models have been successfully generated for several of the single gene disorders [53-56] and can be used for effective drug screening. Thus, specific therapy is likely to emerge sooner for these genetically defined forms of ASD [54].

Collectively, known genetic causes of ASD are observed in around 20\% of ASD patients [2]. Yet each of these genetic changes is rare and only accounts for less than $2 \%$ of cases [2]. What are the genetic changes underlying the high heritability in the rest of the $80 \%$ of ASD patients? This question has been the focus of ASD genetics research over the last decade, which demonstrated that the clinical heterogeneity of ASD is mirrored by an equally daunting genetic heterogeneity $[2,16]$. A combination of common genetic variants and rare mutations is currently believed to underlie ASD heritability. It has been proposed that genetic changes in many genes, estimated in the hundreds, are necessary in order for the disease to occur $[16,57-62]$. Thus, to advance toward estimating the genetic risk of ASD before symptomatology occurs, it appears necessary to develop genetic tests that simultaneously take into account multiple genetic markers, and perhaps multiple types of biological markers.

In concordance with this model, Skafidas and colleagues used genome-wide SNP data in order to build a diagnostic classifier [63]. In this study, the genotyping data for 975 ASD cases and their unaffected relatives were used to identify pathways associated with the disease using a set enrichment analysis. The genes included in the 13 significant pathways identified contained 775 unique SNPs. Of these, 237 SNPs were determined to be highly significant and were used as a diagnostic classifier, applied on a training cohort and two independent validation cohorts. The classifier reached $84 \%$ diagnostic prediction accuracy in a cohort ethnically similar to the one used to build the classifier but was suboptimal for a cohort of ethnically dissimilar individuals (prediction accuracy of 56\%).

A recent study [64] attempted to use pattern classification based on SNP markers and brain imaging markers (regional thickness and regional volume) in order to discriminate between Asperger syndrome and high-functioning autism patients. It would be conceptually interesting to try to incorporate these distinct types of measurements into a single classifier. However, the study only genotyped SNPs in 8 ASD susceptibility genes rather than genome-wide and analyzed the SNP and imaging data separately, comparing their performance for diagnostic classification. In this particular study, SNP genotyping was superior to brain imaging in terms of classification accuracy, but the number of subjects (15 highfunctioning autism and 3 Asperger syndrome) was too small for the results to be generalized.

Future studies combining common sequence variants and rare genetic variation are warranted for identifying panels of genetic markers with high ASD predictive value.

\section{Gene Expression Biomarkers}

Unlike genetic markers, which are variations in DNA sequence and are largely invariable across tissues and during an individual's life, the amount of RNA transcribed from each gene is tissue specific and varies in response to environmental changes. Thus, gene expression levels represent a functional readout of DNA sequence. In a search for biomarkers for ASD, several groups have investigated gene expression profiles of readily available peripheral tissues (i.e., blood and lymphoblasts) from ASD patients [65-70]. Notably, the selection criteria for the ASD group varied markedly between studies and consequently so did the gene expression signatures identified (reviewed in $[70,71]$ ). However, a common theme of these studies was the upregulation of genes involved in immune and inflammatory responses, consistent with gene expression studies on postmortem brain $[72,73]$ and neuropathological studies $[74,75]$.

A recent study attempted to build a diagnostic classifier using microarray expression profiling of peripheral blood from infants and toddlers with ASD [76]. Out of an initial set of differentially expressed probes, 48 were selected as an optimal classifier by applying a support vector machine (SVM) algorithm to half of the microarray dataset. The accuracy of this classifier in correctly diagnosing ASD cases from the second half of the dataset was $91 \%$. However, the validation dataset was not independently generated; so, the performance of this classifier remains to be replicated.

The largest study on blood gene expression profiling in ASD patients to date [77] used two independently generated datasets: one consisting of genome-wide expression profiles from 66 ASD males and 33 male controls and another set of data from 104 ASD cases and 82 controls. The mean age for subjects in this study was 8-9 years. Based on genes differentially expressed in the first dataset, the authors generated a set of 55 probes that had the highest accuracy in discriminating between cases and controls. Using this set 
of 55 genes, the accuracy of diagnostic classification in the second dataset was $67.7 \%$. As expected from the fact that the first dataset contained only males, the classifier accuracy in the second dataset was higher for males than females. It is worth noting that the two classifiers described previously are entirely distinct in their composition of genes. This may be explained at least partially by the fact that the two studies looked at different age groups.

Overall, gene expression measurements in peripheral tissues from ASD patients are still far from achieving diagnostic accuracy. Subphenotyping, detailed clinical characterization of study subjects, and combining genome-wide expression profiles with data on DNA sequence variation and/or epigenetic modifications would be needed in order to increase the power of detecting disease-relevant gene expression changes in peripheral tissues.

\section{Biomarkers of Altered Immune Responses}

Mounting evidence suggests that ASD is associated with abnormalities in the innate and adaptive immune responses (reviewed in [78]). Increased levels of plasma interleukins IL-1, IL-6, IL-8, and IL-12, interferon $\gamma$, and macrophage migration inhibitory factor, as well as decreased levels of TGF- $\beta$, have been described in ASD patients [79-82]. In addition, ASD patients were reported to have increased levels of plasma IgG immunoglobulin and abnormal activation of natural killer cells $[65,83,84]$. Autoantibodies against neural cells and brain tissue were also detected in the plasma of ASD children [85-89]. Postmortem brain studies have demonstrated an increase in microglial activation in ASD patients, and this observation has recently been confirmed by positron emission tomography in adults with ASD using a radiotracer for microglia [74, 75, 90]. Suzuki et al. [91] observed an increased signal for activated microglia in cerebellum, midbrain, pons, fusiform gyri, anterior cingulate, and orbitofrontal cortex in ASD subjects. Recently, Momeni et al. [92] used surface-enhanced laser desorption/ionization time-of-flight (SELDI TOF) mass spectrometry to identify potential peptide biomarkers in the plasma of ASD children. The study identified three related complement $\mathrm{C} 3$ peptides differentially expressed between ASD and control children. Although no validation dataset was available, the study highlighted the potential of proteomic approaches to detect immune biomarkers for ASD.

It is not entirely clear if the immunological changes observed in ASD patients are causally implicated in the disease, but at least some of the abnormal immune changes appear to contribute to behavioral changes. For example, mouse studies have shown that maternal immune activation during pregnancy leads to ASD-like behaviors in offspring [78], and the increase in inactivated immune cells in ASD brain may lead to altered synaptic plasticity [93].

The immune mechanisms in ASD are a promising research avenue that may result in targeted therapy, and thus, further studies are needed to identify reliable immune markers that can define the group of ASD patients likely to benefit from treatments targeting immune responses.

\section{Other Types of Biomarkers}

8.1. Head Circumference. Head circumference is one of the most extensively investigated early biological markers of autism, used as a proxy for brain size. The increased head circumference in autistic children was one of the clinical characteristics described by Kanner [94] and was further assessed by multiple studies (reviewed in [95]).

Although there was some variability between the results of various groups, the overall conclusion of earlier studies, based on small samples of less than 100 individuals, was that at birth, the head circumference of children who are later diagnosed with ASD is normal or smaller than normal [95]. During the first three years of life, however, autism appeared to be associated with an accelerated rate of head growth leading to macrocephaly, that is, a head circumference more than two standard deviations above the population mean [95]. The exact time window when the increased head growth occurs is still debated. For example, one study observed that the increased head growth rate was limited to the first year of life [96], while another group found no increase in head growth rate during the first year of life but did observe an increase in the rate of overall body growth [97]. Rommelse and colleagues noted that although the head circumference of autistic children was higher than the population norm, the same was true for children with other psychiatric disorders [98]. This study thus concluded that increased head growth is a characteristic of psychiatric disorders in general, rather than being specific for ASD.

A recent multicenter study examined the head circumference in a sample of 9000 children, $1 \%$ of whom were diagnosed with autism [99]. Barnard-Brak et al. observed no difference in head circumference between autistic and nonautistic children and no difference in the incidence of macrocephaly between the autism and control groups. The authors suggested that the difference between their results and previous studies may result from selection bias affecting clinic-based, small cohort studies. Thus, it is possible that a subgroup of autistic children may be characterized by macrocephaly, but the observation does not appear to be generalizable to the wide ASD spectrum. Notably, head circumference is a heritable trait in the general population, and Froehlich and colleagues recently reported an increased incidence of macrocephaly in the group of autistic children studied, as well as in their unaffected twins [100].

8.2. Serotonin. Hyperserotonemia is one of the first blood biomarkers to have been implicated in ASD. Increased levels of serotonin in whole blood are consistently observed in 25$35 \%$ of ASD patients [101, 102]. Serotonin levels have been shown to be heritable and regulated by genetic variants in the serotonin receptor gene SLC6A4 and the integrin beta gene ITGB3 $[103,104]$. Interestingly, a recent study describing a mouse model carrying an SLC6A4 variant [105] showed hyperserotonemia and behavioral changes including social deficits and repetitive behaviors, suggesting that the serotonin imbalance may causally contribute to ASD, and offering hope that therapies targeting the serotonin pathway may prove beneficial for a subset of ASD patients. 
A number of other soluble brain biomarkers such as VIP, substance-P, NGF, BDNF, and secretin have been reported in ASD (reviewed in [22]) and await replication in independent studies.

8.3. Mitochondrial and Metabolic Markers. Mitochondrial disease (MD) has been reported to occur with higher frequency among ASD patients than in the general population $[106,107]$. In addition to mitochondrial disease being diagnosed more frequently among ASD cases, biochemical markers of mitochondrial function are also altered in ASD patients without MD $[108,109]$. Thus, it has been proposed that ASD with mitochondrial dysfunction may represent a phenotypically distinct subgroup of ASD. A meta-analysis of mitochondrial dysfunction in ASD noted that many of the studies implicating MD in ASD are based on small cohorts or single case reports [110], and thus, further research is warranted for establishing the value of biomarkers of mitochondrial dysfunction in ASD.

Changes in porphyrin metabolism have been associated with ASD [111, 112] and are believed to reflect exposure to environmental toxins. Heyer et al. [113] investigated the urine levels of pentapophyrin and coproporphyrin as potential markers for ASD risk by comparing a group of 30 male children with autistic disorder, 14 with PDD-NOS, and 32 neurotypical controls. ASD children (including PDD-NOS and autism) had higher urinary levels of pentaporphyrin and coproporphyrin compared to age matched controls. The sensitivity of urinary pentaporphyrin was 30\% for autism and $36 \%$ for PDD-NOS, and the sensitivity of coproporphyrin was $33 \%$ for autism and 14\% for PDD-NOS; both makers reached 94\% specificity for ASD in this study.

Multivariate analyses of blood and urine proteins and metabolites have also been attempted in search for ASD biomarkers. Yap and colleagues [114] used NMR spectroscopy to measure urine metabolites in 39 ASD children and their unaffected siblings. This study reported higher levels of urinary taurine and lower levels of urinary glutamate in ASD children, as well as metabolic changes consistent with abnormalities in gut microbiota. Schwarz et al. [20] performed immunoassays of 147 analytes using blood serum from 45 adult subjects with Asperger syndrome (AS) and 50 controls. The AS and control groups were divided into a discovery and validation group for the male and female subjects, respectively. A panel of 9 analytes were found to be significantly different between male AS cases and male controls in the male discovery group. Applying this panel as classifier to the male validation group resulted in correct classification of $70 \%$ of AS males in the validation group but was inefficient at discriminating between female AS and female controls. Similarly, the panel of 14 biomarkers identified as significantly different between female AS and female controls was able to correctly classify $90 \%$ of the AS females in the validation group but did not discriminate between male AS and male controls.

8.4. Biomarkers of Oxidative Stress. Oxidative stress (OS) results from insufficient counteracting of endogenous and exogenous reactive oxygen species (ROS), as a result of either ineffective antioxidant mechanisms, excessive production of ROS, or both. Evidence of OS in ASD has been reported by numerous studies of blood and brain OS biomarkers. Decreased plasma levels of reduced glutathione, glutathione peroxidase, methionine, and cysteine and increased plasma levels of oxidized glutathione have been reported in ASD subjects [115-117]. Measurements of OS biomarkers in postmortem brain tissue from ASD cases [118] demonstrated changes in reduced and oxidized glutathione and increased levels of 3-nitrotyrosine and 8-oxo-deoxyguanosine, which are markers of oxidative protein damage. A meta-analysis of OS biomarkers in ASD [115] showed that the strongest differences between ASD cases and controls in the mean OS biomarker levels were observed for reduced glutathione (decreased by 27\%) and oxidized glutathione (increased by $45 \%)$. This meta-analysis study also highlighted the fact that OS biomarker changes associated with ASD tend to be heterogeneous, and the observations are based on small sample sizes and moderate effects, thus cautioning on the need for further standardized studies.

\section{Conclusions and Ethical Considerations}

Although many avenues have been tried for identifying biological markers for ASD, a clinically valuable ASD biomarker is not yet in sight. For a biomarker to become clinically valuable, it would need to be highly sensitive and specific (even if limited to a well-defined subgroup of ASD patients or to a developmental window), be feasible for use in the clinic, and not be cost prohibitive. The majority of studies currently available suffer from small cohort sizes and lack of replication in independent datasets, which make the estimation of biomarker reliability hard to evaluate. The difficulty of putting together a large ASD research cohort may be balanced out in the near future by more open data sharing, allowing investigators to replicate their results using published data.

As the development of ASD biomarkers is still in its infancy, there are valid concerns among clinicians that premature translation of research data into commercially available tests may be harmful rather than beneficial for ASD patients and their families [119]. The majority of genetic risk factors identified thus far have small effect sizes (i.e., they only marginally increase the disease risk over the population standard). Thus, it is questionable whether disclosing the result of such an ASD risk marker to the family is in fact beneficial. The results of genetic biomarker tests are likely to have a huge impact on parental decision making for reproduction, and thus, more research may be needed for better understanding parental needs and attitudes [11]. In addition, communicating the results of a risk biomarker should ensure that children do not receive a disease label that will affect their future options and potential.

To properly control the translation of research results to the clinic, it has been proposed [120] that assessing ASD genetic research should follow a similar process to the ACCE Model Project established by the Office of Public Health 
Genomics (OPHG) of Center for Disease Control, which provides an analytic framework to evaluate the analytic validity, clinical validity, clinical utility, and associated ethical, legal, and social implications of genetic tests [121]. Similar analytical frameworks would be very valuable for all classes of ASD biomarkers.

The lack of effective biomarkers for ASD despite progressive accumulation of research data may seem daunting, but as the field matures and incorporates a deeper understanding of disease heterogeneity into study designs and analytical methods, the translation of biomarker research to clinic may eventually become within reach.

\section{Acknowledgments}

This work was supported by a NARSAD Young Investigator Award (I. Voineagu), a grant-in-aid from the Japanese Society for Promotion of Science (I. Voineagu), and a Korea Healthcare Technology R\&D Project (A120029) from the Ministry of Health and Welfare, Republic of Korea (H. J. Yoo).

\section{References}

[1] S. H. Kim and C. Lord, "The behavioral manifestations of autism spectrum disorders," in The Neuroscience of autism Spectrum Disorders, pp. 25-37, 2013.

[2] B. S. Abrahams and D. H. Geschwind, "Advances in autism genetics: on the threshold of a new neurobiology," Nature Reviews Genetics, vol. 9, no. 5, pp. 341-355, 2008.

[3] A. P. Association, Diagnostic and Statistical Manual of Mental Disorders: DSM-IV, American Psychiatric Association, Washington, DC, USA, 2000.

[4] M. Elsabbagh, G. Divan, Y. J. Koh et al., "Global prevalence of autism and other pervasive developmental disorders," Autism Research, vol. 5, no. 3, pp. 160-179, 2012.

[5] C. Lord, E. Petkova, V. Hus et al., "A multisite study of the clinical diagnosis of different autism spectrum disorders," Archives of General Psychiatry, vol. 69, no. 3, pp. 306-313, 2012.

[6] D. H. Skuse, "DSM-5's conceptualization of autistic disorders," Journal of the American Academy of Child \& Adolescent Psychiatry, vol. 51, no. 4, pp. 344-346, 2012.

[7] J. C. McPartland, B. Reichow, and F. R. Volkmar, "Sensitivity and specificity of proposed DSM-5 diagnostic criteria for autism spectrum disorder," Journal of the American Academy of Child and Adolescent Psychiatry, vol. 51, no. 4, pp. 368-383, 2012.

[8] C. Ecker, W. Spooren, and D. G. Murphy, "Translational approaches to the biology of Autism: false dawn or a new era?" Molecular Psychiatry, vol. 18, no. 4, pp. 435-442, 2013.

[9] L. Kanner, "The original description of autism, with excellent case examples," Nervous Child, vol. 2, pp. 217-250, 1943.

[10] J. Atkinson, W. A. Colburn, V. G. DeGruttola et al., "Biomarkers and surrogate endpoints: preferred definitions and conceptual framework," Clinical Pharmacology and Therapeutics, vol. 69, no. 3, pp. 89-95, 2001.

[11] P. Walsh, M. Elsabbagh, P. Bolton, and I. Singh, "In search of biomarkers for autism: scientific, social and ethical challenges," Nature Reviews Neuroscience, vol. 12, no. 10, pp. 603-612, 2011.

[12] E. Ben Itzchak, E. Lahat, R. Burgin, and A. D. Zachor, "Cognitive, behavior and intervention outcome in young children with autism," Research in Developmental Disabilities, vol. 29, no. 5, pp. 447-458, 2008.

[13] G. Dawson, S. Rogers, J. Munson et al., "Randomized, controlled trial of an intervention for toddlers with autism: the early start Denver model," Pediatrics, vol. 125, no. 1, pp. e17-e23, 2010.

[14] S. J. Rogers and L. A. Vismara, "Evidence-based comprehensive treatments for early autism," Journal of Clinical Child and Adolescent Psychology, vol. 37, no. 1, pp. 8-38, 2008.

[15] L. D. Wiggins, J. Baio, and C. Rice, "Examination of the time between first evaluation and first autism spectrum diagnosis in a population-based sample," Journal of Developmental and Behavioral Pediatrics, vol. 27, supplement 2, pp. S79-S87, 2006.

[16] M. W. State and P. Levitt, "The conundrums of understanding genetic risks for autism spectrum disorders," Nature Neuroscience, vol. 14, no. 12, pp. 1499-1506, 2011.

[17] R. M. Cantor, "Molecular genetics of autism," Current Psychiatry Reports, vol. 11, no. 2, pp. 137-142, 2009.

[18] V. W. Hu, "Subphenotype-dependent disease markers for diagnosis and personalized treatment of autism spectrum disorders," Disease Markers, vol. 33, no. 5, pp. 277-288, 2012.

[19] D. M. Werling and D. H. Geschwind, "Sex differences in autism spectrum disorders," Current Opinion in Neurology, vol. 26, no. 2, pp. 146-153, 2013.

[20] E. Schwarz, P. C. Guest, H. Rahmoune et al., "Sex-specific serum biomarker patterns in adults with Asperger's syndrome," Molecular Psychiatry, vol. 16, no. 12, pp. 1213-1220, 2011.

[21] J. M. Lattin, J. D. Carroll, and P. E. Green, Analyzing Multivariate Data, Thomson Brooks/Cole, Pacific Grove, Calif, USA, 2003.

[22] H. V. Ratajczak, "Theoretical aspects of autism: biomarkersa review," Journal of Immunotoxicology, vol. 8, no. 1, pp. 80-94, 2011.

[23] K. A. Stigler and C. J. McDougley, "Structural and functional MRI studies of autism spectrum disorders," in The Neuroscience of autism Spectrum Disorders, pp. 251-266, 2013.

[24] R. A. Carper, P. Moses, Z. D. Tigue, and E. Courchesne, "Cerebral lobes in autism: early hyperplasia and abnormal age effects," NeuroImage, vol. 16, no. 4, pp. 1038-1051, 2002.

[25] C. C. Brun, R. Nicolson, N. Leporé et al., "Mapping brain abnormalities in boys with autism," Human Brain Mapping, vol. 30, no. 12, pp. 3887-3900, 2009.

[26] S. J. M. C. Palmen, H. E. Hulshoff Pol, C. Kemner et al., "Increased gray-matter volume in medication-naive highfunctioning children with autism spectrum disorder," Psychological Medicine, vol. 35, no. 4, pp. 561-570, 2005.

[27] C. Ecker, A. Marquand, J. Mourão-Miranda et al., "Describing the brain in autism in five dimensions-magnetic resonance imaging-assisted diagnosis of autism spectrum disorder using a multiparameter classification approach," Journal of Neuroscience, vol. 30, no. 32, pp. 10612-10623, 2010.

[28] L. Q. Uddin, V. Menon, C. B. Young et al., "Multivariate searchlight classification of structural magnetic resonance imaging in children and adolescents with autism," Biological Psychiatry, vol. 70, no. 9, pp. 833-841, 2011.

[29] M. Ingalhalikar, D. Parker, L. Bloy, T. P. L. Roberts, and R. Verma, "Diffusion based abnormality markers of pathology: toward learned diagnostic prediction of ASD," NeuroImage, vol. 57, no. 3, pp. 918-927, 2011.

[30] N. Lange, M. B. Dubray, J. E. Lee et al., "Atypical diffusion tensor hemispheric asymmetry in autism," Autism Research, vol. 3, no. 6, pp. 350-358, 2010. 
[31] N. J. Minshew and T. A. Keller, "The nature of brain dysfunction in autism: functional brain imaging studies," Current Opinion in Neurology, vol. 23, no. 2, pp. 124-130, 2010.

[32] K. Pierce, R.-A. Müller, J. Ambrose, G. Allen, and E. Courchesne, "Face processing occurs outside the fusiform 'face area' in autism: evidence from functional MRI," Brain, vol. 124, no. 10, pp. 2059-2073, 2001.

[33] R. T. Schultz, I. Gauthier, A. Klin et al., "Abnormal ventral temporal cortical activity during face discrimination among individuals with autism and Asperger syndrome," Archives of General Psychiatry, vol. 57, no. 4, pp. 331-340, 2000.

[34] D. Hubl, S. Bölte, S. Feineis-Matthews et al., "Functional imbalance of visual pathways indicates alternative face processing strategies in autism," Neurology, vol. 61, no. 9, pp. 1232-1237, 2003.

[35] B. A. Corbett, V. Carmean, S. Ravizza et al., "A functional and structural study of emotion and face processing in children with autism," Psychiatry Research, vol. 173, no. 3, pp. 196-205, 2009.

[36] K. A. Pelphrey, J. P. Morris, G. Mccarthy, and K. S. Labar, "Perception of dynamic changes in facial affect and identity in autism," Social Cognitive and Affective Neuroscience, vol. 2, no. 2, pp. 140-149, 2007.

[37] M. Dapretto, M. S. Davies, J. H. Pfeifer et al., "Understanding emotions in others: mirror neuron dysfunction in children with autism spectrum disorders," Nature Neuroscience, vol. 9, no. 1, pp. 28-30, 2006.

[38] E. Redcay and E. Courchesne, "Deviant functional magnetic resonance imaging patterns of brain activity to speech in 23 -year-old children with autism spectrum disorder," Biological Psychiatry, vol. 64, no. 7, pp. 589-598, 2008.

[39] D. P. Kennedy and E. Courchesne, "The intrinsic functional organization of the brain is altered in autism," NeuroImage, vol. 39, no. 4, pp. 1877-1885, 2008.

[40] C. S. Monk, S. J. Peltier, J. L. Wiggins et al., "Abnormalities of intrinsic functional connectivity in autism spectrum disorders," NeuroImage, vol. 47, no. 2, pp. 764-772, 2009.

[41] J. S. Anderson, J. A. Nielsen, A. L. Froehlich et al., "Functional connectivity magnetic resonance imaging classification of autism," Brain, vol. 134, no. 12, pp. 3742-3754, 2011.

[42] W. Bosl, A. Tierney, H. Tager-Flusberg, and C. Nelson, "EEG complexity as a biomarker for autism spectrum disorder risk," BMC Medicine, vol. 9, article 18, 2011.

[43] G. Dawson, E. J. H. Jones, K. Merkle et al., "Early behavioral intervention is associated with normalized brain activity in young children with autism," Journal of the American Academy of Child \& Adolescent Psychiatry, vol. 51, no. 11, pp. 1150-1159, 2012.

[44] T. P. L. Roberts, S. Y. Khan, M. Rey et al., "MEG detection of delayed auditory evoked responses in autism spectrum disorders: towards an imaging biomarker for autism," Autism Research, vol. 3, no. 1, pp. 8-18, 2010.

[45] S. J. Webb, G. Dawson, R. Bernier, and H. Panagiotides, "ERP evidence of atypical face processing in young children with autism," Journal of autism and Developmental Disorders, vol. 36, no. 7, pp. 881-890, 2006.

[46] R. Griffin and C. Westbury, "Infant EEG activity as a biomarker for autism: a promising approach or a false promise?" BMC Medicine, vol. 9, article 61, 2011.

[47] A. Bailey, A. Le Couteur, I. Gottesman et al., "Autism as a strongly genetic disorder: evidence from a British twin study," Psychological Medicine, vol. 25, no. 1, pp. 63-77, 1995.
[48] S. Steffenburg, C. Gillberg, L. Hellgren et al., "A twin study of autism in Denmark, Finland, Iceland, Norway and Sweden," Journal of Child Psychology and Psychiatry and Allied Disciplines, vol. 30, no. 3, pp. 405-416, 1989.

[49] J. Hallmayer, S. Cleveland, A. Torres et al., "Genetic heritability and shared environmental factors among twin pairs with autism," Archives of General Psychiatry, vol. 68, no. 11, pp. 10951102, 2011.

[50] A. L. Beaudet, "The utility of chromosomal microarray analysis in developmental and behavioral pediatrics," Child Development, vol. 84, no. 1, pp. 121-132, 2013.

[51] K. M. Heil and C. P. Schaaf, "The genetics of autism spectrum disorders-a guide for clinicians," Current Psychiatry Reports, vol. 15, no. 1, article 334, 2013.

[52] D. T. Miller, M. P. Adam, S. Aradhya et al., "Consensus statement: chromosomal microarray is a first-tier clinical diagnostic test for individuals with developmental disabilities or congenital anomalies," American Journal of Human Genetics, vol. 86, no. 5, pp. 749-764, 2010.

[53] H.-T. Chao, H. Chen, R. C. Samaco et al., "Dysfunction in GABA signalling mediates autism-like stereotypies and Rett syndrome phenotypes," Nature, vol. 468, no. 7321, pp. 263-269, 2010.

[54] G. Dolen, E. Osterweil, B. S. Shankaranarayana Rao et al., "Correction of fragile X syndrome in mice," Neuron, vol. 56, no. 6, pp. 955-962, 2007.

[55] O. Peñagarikano, B. S. Abrahams, E. I. Herman et al., "Absence of CNTNAP2 leads to epilepsy, neuronal migration abnormalities, and core autism-related deficits," Cell, vol. 147, no. 1, pp. 235-246, 2011.

[56] S. E. P. Smith, Y.-D. Zhou, G. Zhang, Z. Jin, D. C. Stoppel, and M. P. Anderson, "Increased gene dosage of Ube3a results in autism traits and decreased glutamate synaptic transmission in mice," Science Translational Medicine, vol. 3, no. 103, article 103ra97, 2011.

[57] M. W. State, "The genetics of child psychiatric disorders: focus on autism and tourette syndrome," Neuron, vol. 68, no. 2, pp. 254-269, 2010.

[58] B. M. Neale, Y. Kou, L. Liu et al., "Patterns and rates of exonic de novo mutations in autism spectrum disorders," Nature, vol. 485, no. 7397, pp. 242-245, 2012.

[59] B. J. O’Roak, L. Vives, S. Girirajan et al., "Sporadic autism exomes reveal a highly interconnected protein network of de novo mutations," Nature, vol. 485, no. 7397, pp. 246-250, 2012.

[60] S. J. Sanders, M. T. Murtha, A. R. Gupta et al., "De novo mutations revealed by whole-exome sequencing are strongly associated with autism," Nature, vol. 485, no. 7397, pp. 237-241, 2012.

[61] E. T. Lim, S. Raychaudhuri, S. J. Sanders et al., "Rare complete knockouts in humans: population distribution and significant role in autism spectrum disorders," Neuron, vol. 77, no. 2, pp. 235-242, 2013.

[62] T. W. Yu, M. H. Chahrour, M. E. Coulter et al., "Using wholeexome sequencing to identify inherited causes of autism," Neuron, vol. 77, no. 2, pp. 259-273, 2013.

[63] E. Skafidas, R. Testa, D. Zantomio, G. Chana, I. P. Everall, and C. Pantelis, "Predicting the diagnosis of autism spectrum disorder using gene pathway analysis," Molecular Psychiatry, 2012.

[64] Y. Jiao, R. Chen, X. Ke et al., "Predictive models for subtypes of autism spectrum disorder based on single-nucleotide polymorphisms and magnetic resonance imaging," Advances in Medical Sciences, vol. 56, no. 2, pp. 334-342, 2011. 
[65] A. M. Enstrom, L. Lit, C. E. Onore et al., "Altered gene expression and function of peripheral blood natural killer cells in children with autism," Brain, Behavior, and Immunity, vol. 23, no. 1, pp. 124-133, 2009.

[66] J. P. Gregg, L. Lit, C. A. Baron et al., "Gene expression changes in children with autism," Genomics, vol. 91, no. 1, pp. 22-29, 2008.

[67] V. W. Hu, B. C. Frank, S. Heine, N. H. Lee, and J. Quackenbush, "Gene expression profiling of lymphoblastoid cell lines from monozygotic twins discordant in severity of autism reveals differential regulation of neurologically relevant genes," $B M C$ Genomics, vol. 7, article 118, 2006.

[68] V. W. Hu, A. T. Nguyen, K. S. Kim et al., "Gene expression profiling of lymphoblasts from autistic and nonaffected sib pairs: altered pathways in neuronal development and steroid biosynthesis," PLoS ONE, vol. 4, no. 6, article e5775, 2009.

[69] V. W. Hu and M. E. Steinberg, "Novel clustering of items from the autism diagnostic interview-revised to define phenotypes within autism spectrum disorders," Autism Research, vol. 2, no. 2, pp. 67-77, 2009.

[70] C. Lintas, R. Sacco, and A. M. Persico, "Genome-wide expression studies in autism spectrum disorder, Rett syndrome, and Down syndrome," Neurobiology of Disease, vol. 45, no. 1, pp. 5768, 2012.

[71] I. Voineagu, "Gene expression studies in autism: moving from the genome to the transcriptome and beyond," Neurobiology of Disease, vol. 45, no. 1, pp. 69-75, 2012.

[72] K. Garbett, P. J. Ebert, A. Mitchell et al., "Immune transcriptome alterations in the temporal cortex of subjects with autism," Neurobiology of Disease, vol. 30, no. 3, pp. 303-311, 2008.

[73] I. Voineagu, X. Wang, P. Johnston et al., "Transcriptomic analysis of autistic brain reveals convergent molecular pathology," Nature, vol. 474, no. 7351, pp. 380-386, 2011.

[74] J. T. Morgan, G. Chana, C. A. Pardo et al., "Microglial activation and increased microglial density observed in the dorsolateral prefrontal cortex in autism," Biological Psychiatry, vol. 68, no. 4, pp. 368-376, 2010.

[75] D. L. Vargas, C. Nascimbene, C. Krishnan, A. W. Zimmerman, and C. A. Pardo, "Neuroglial activation and neuroinflammation in the brain of patients with autism," Annals of Neurology, vol. 57, no. 1, pp. 67-81, 2005.

[76] S. J. Glatt, M. T. Tsuang, M. Winn et al., "Blood-based gene expression signatures of infants and toddlers with autism," Journal of the American Academy of Child \& Adolescent Psychiatry, vol. 51, no. 9, pp. 934.e2-944.e2, 2012.

[77] S. W. Kong, C. D. Collins, Y. Shimizu-Motohashi et al., "Characteristics and predictive value of blood transcriptome signature in males with autism spectrum disorders," PLoS One, vol. 7, no. 12, article e49475, 2012.

[78] C. Onore, M. Careaga, and P. Ashwood, "The role of immune dysfunction in the pathophysiology of autism," Brain, Behavior, and Immunity, vol. 26, no. 3, pp. 383-392, 2012.

[79] P. Ashwood, A. Enstrom, P. Krakowiak et al., "Decreased transforming growth factor betal in autism: a potential link between immune dysregulation and impairment in clinical behavioral outcomes," Journal of Neuroimmunology, vol. 204, no. 1-2, pp. 149-153, 2008.

[80] P. Ashwood, P. Krakowiak, I. Hertz-Picciotto, R. Hansen, I. Pessah, and J. Van de Water, "Elevated plasma cytokines in autism spectrum disorders provide evidence of immune dysfunction and are associated with impaired behavioral outcome," Brain, Behavior, and Immunity, vol. 25, no. 1, pp. 40-45, 2011.
[81] E. L. Grigorenko, S. S. Han, C. M. Yrigollen et al., "Macrophage migration inhibitory factor and autism spectrum disorders," Pediatrics, vol. 122, no. 2, pp. e438-e445, 2008.

[82] K. Okada, K. Hashimoto, Y. Iwata et al., "Decreased serum levels of transforming growth factor-betal in patients with autism," Progress in Neuro-Psychopharmacology and Biological Psychiatry, vol. 31, no. 1, pp. 187-190, 2007.

[83] J. Croonenberghs, A. Wauters, K. Devreese et al., "Increased serum albumin, $\gamma$ globulin, immunoglobulin IgG, and IgG2 and IgG4 in autism," Psychological Medicine, vol. 32, no. 8, pp. 14571463, 2002.

[84] C. A. Molloy, A. L. Morrow, J. Meinzen-Derr et al., "Elevated cytokine levels in children with autism spectrum disorder," Journal of Neuroimmunology, vol. 172, no. 1-2, pp. 198-205, 2006.

[85] A. M. Connolly, M. Chez, E. M. Streif et al., "Brain-derived neurotrophic factor and autoantibodies to neural antigens in sera of children with autistic spectrum disorders, LandauKleffner syndrome, and epilepsy," Biological Psychiatry, vol. 59, no. 4, pp. 354-363, 2006.

[86] P. Goines, L. Haapanen, R. Boyce et al., "Autoantibodies to cerebellum in children with autism associate with behavior," Brain, Behavior, and Immunity, vol. 25, no. 3, pp. 514-523, 2011.

[87] C. C. Rossi, J. van de Water, S. J. Rogers, and D. G. Amaral, "Detection of plasma autoantibodies to brain tissue in young children with and without autism spectrum disorders," Brain, Behavior, and Immunity, vol. 25, no. 6, pp. 1123-1135, 2011.

[88] S. Wills, M. Cabanlit, J. Bennett, P. Ashwood, D. Amaral, and J. van de Water, "Autoantibodies in autism Spectrum Disorders (ASD)," Annals of the New York Academy of Sciences, vol. 1107, pp. 79-91, 2007.

[89] S. Wills, M. Cabanlit, J. Bennett, P. Ashwood, D. G. Amaral, and J. Van de Water, "Detection of autoantibodies to neural cells of the cerebellum in the plasma of subjects with autism spectrum disorders," Brain, Behavior, and Immunity, vol. 23, no. 1, pp. 6474, 2009.

[90] T. C. Theoharides, S. Asadi, and A. B. Patel, "Focal brain inflammation and autism," Journal of Neuroinflammation, vol. 10, article 46, 2013.

[91] K. Suzuki, G. Sugihara, Y. Ouchi et al., "Microglial activation in young adults with autism spectrum disorder," JAMA Psychiatry, vol. 70, no. 1, pp. 49-58, 2013.

[92] N. Momeni, J. Bergquist, L. Brudin et al., "A novel bloodbased biomarker for detection of autism spectrum disorders," Translational Psychiatry, vol. 2, article e91, 2012.

[93] L. M. Boulanger, "Immune proteins in brain development and synaptic plasticity," Neuron, vol. 64, no. 1, pp. 93-109, 2009.

[94] L. Kanner, "Autistic disturbances of affective contact," Acta Paedopsychiatrica, vol. 35, no. 4, pp. 100-136, 1968.

[95] M. L. Barton et al., "Early manifestations of autism spectrum disorders," in The Neuroscience of autism Spectrum Disorders, pp. 39-53, 2013.

[96] G. Dawson, "Early behavioral intervention, brain plasticity, and the prevention of autism spectrum disorder," Development and Psychopathology, vol. 20, no. 3, pp. 775-803, 2008.

[97] E. van Daalen, S. H. N. Swinkels, C. Dietz, H. van Engeland, and J. K. Buitelaar, "Body length and head growth in the first year of life in Autism," Pediatric Neurology, vol. 37, no. 5, pp. 324-330, 2007.

[98] N. N. J. Rommelse, C. T. R. Peters, I. J. Oosterling et al., "A pilot study of abnormal growth in autism spectrum disorders and other childhood psychiatric disorders," Journal of autism and Developmental Disorders, vol. 41, no. 1, pp. 44-54, 2011. 
[99] L. Barnard-Brak, T. Sulak, and J. K. Ivey Hatz, "Macrocephaly in children with autism spectrum disorders," Pediatric Neurology, vol. 44, no. 2, pp. 97-100, 2011.

[100] W. Froehlich, S. Cleveland, A. Torres et al., "Head circumferences in twins with and without autism spectrum disorders," Journal of autism and Developmental Disorders, 2013.

[101] E. J. Mulder, G. M. Anderson, I. P. Kema et al., "Platelet serotonin levels in pervasive developmental disorders and mental retardation: diagnostic group differences, within-group distribution, and behavioral correlates," Journal of the American Academy of Child and Adolescent Psychiatry, vol. 43, no. 4, pp. 491-499, 2004.

[102] R. J. Schain and D. X. Freedman, "Studies on 5-hydroxyindole metabolism in autistic and other mentally retarded children," The Journal of Pediatrics, vol. 58, no. 3, pp. 315-320, 1961.

[103] M. Abney, M. S. McPeek, and C. Ober, "Broad and narrow heritabilities of quantitative traits in a founder population," American Journal of Human Genetics, vol. 68, no. 5, pp. 13021307, 2001.

[104] L. A. Weiss, M. Abney, E. H. Cook Jr., and C. Ober, "Sex-specific genetic architecture of whole blood serotonin levels," American Journal of Human Genetics, vol. 76, no. 1, pp. 33-41, 2005.

[105] J. Veenstra-VanderWeele, C. L. Muller, H. Iwamoto et al., "Autism gene variant causes hyperserotonemia, serotonin receptor hypersensitivity, social impairment and repetitive behavior," Proceedings of the National Academy of Sciences of the United States of America, vol. 109, no. 14, pp. 5469-5474, 2012.

[106] D. A. Rossignol and R. E. Frye, "Mitochondrial dysfunction in autism spectrum disorders: a systematic review and metaanalysis," Molecular Psychiatry, vol. 17, no. 3, pp. 290-314, 2012.

[107] J. R. Weissman, R. I. Kelley, M. L. Bauman et al., "Mitochondrial disease in autism spectrum disorder patients: a cohort analysis," PLoS ONE, vol. 3, no. 11, article e3815, 2008.

[108] C. Correia, A. M. Coutinho, L. Diogo et al., "Brief report: high frequency of biochemical markers for mitochondrial dysfunction in autism: no association with the mitochondrial aspartate/glutamate carrier SLC25A12 gene," Journal of autism and Developmental Disorders, vol. 36, no. 8, pp. 1137-1140, 2006.

[109] G. Oliveira, L. Diogo, M. Grazina et al., "Mitochondrial dysfunction in autism spectrum disorders: a population-based study," Developmental Medicine and Child Neurology, vol. 47, no. 3, pp. 185-189, 2005.

[110] D. A. Rossignol and R. E. Frye, "A review of research trends in physiological abnormalities in autism spectrum disorders: immune dysregulation, inflammation, oxidative stress, mitochondrial dysfunction and environmental toxicant exposures," Molecular Psychiatry, vol. 17, no. 4, pp. 389-401, 2012.

[111] D. W. Austin and K. Shandley, "An investigation of porphyrinuria in Australian children with autism," Journal of Toxicology and Environmental Health A, vol. 71, no. 20, pp. 1349-1351, 2008.

[112] J. S. Woods, S. E. Armel, D. I. Fulton et al., "Urinary porphyrin excretion in neurotypical and autistic children," Environmental Health Perspectives, vol. 118, no. 10, pp. 1450-1457, 2010.

[113] N. J. Heyer, D. Echeverria, and J. S. Woods, "Disordered porphyrin metabolism: a potential biological marker for autism risk assessment," Autism Research, vol. 5, no. 2, pp. 84-92, 2012.

[114] I. K. S. Yap, M. Angley, K. A. Veselkov, E. Holmes, J. C. Lindon, and J. K. Nicholson, "Urinary metabolic phenotyping differentiates children with autism from their unaffected siblings and age-matched controls," Journal of Proteome Research, vol. 9, no. 6, pp. 2996-3004, 2010.
[115] A. Frustaci, M. Neri, A. Cesario et al., "Oxidative stress-related biomarkers in autism: systematic review and meta-analyses," Free Radical Biology and Medicine, vol. 52, no. 10, pp. 2128-2141, 2012.

[116] S. J. James, P. Cutler, S. Melnyk et al., "Metabolic biomarkers of increased oxidative stress and impaired methylation capacity in children with autism," American Journal of Clinical Nutrition, vol. 80, no. 6, pp. 1611-1617, 2004.

[117] S. J. James, S. Melnyk, S. Jernigan et al., "Metabolic endophenotype and related genotypes are associated with oxidative stress in children with autism," American Journal of Medical Genetics B: Neuropsychiatric Genetics, vol. 141, no. 8, pp. 947-956, 2006.

[118] S. Rose, S. Melnyk, O. Pavliv et al., "Evidence of oxidative damage and inflammation associated with low glutathione redox status in the autism brain," Translational Psychiatry, vol. 2, article e134, 2012.

[119] B. R. Jordan and D. F. C. Tsai, "Whole-genome association studies for multigenic diseases: ethical dilemmas arising from commercializationd the case of genetic testing for autism," Journal of Medical Ethics, vol. 36, no. 7, pp. 440-444, 2010.

[120] S. W. Scherer and G. Dawson, "Risk factors for autism: translating genomic discoveries into diagnostics," Human Genetics, vol. 130, no. 1, pp. 123-148, 2011.

[121] Center for Disease Control, "Public Health Genomics: Genetic Testing-ACCE Model System for Collecting, Analyzing and Disseminating Information on Genetic Tests," 2010, http://www.cdc.gov/genomics/gtesting/ACCE/FBR/index .htm. 


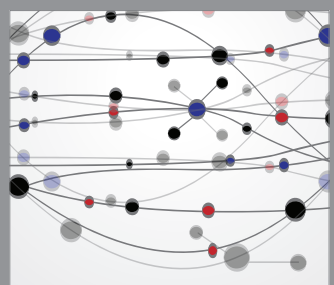

The Scientific World Journal
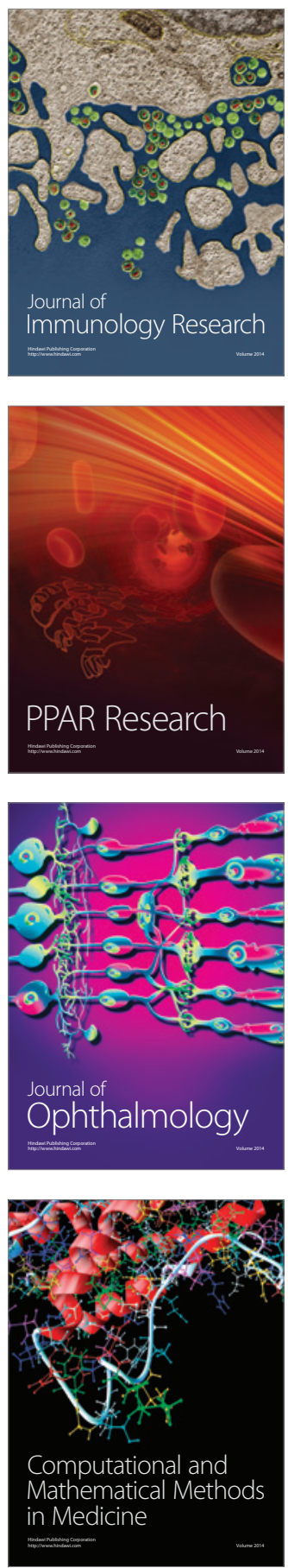

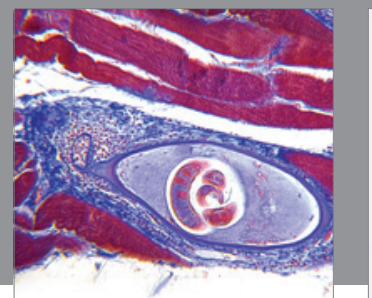

Gastroenterology

Research and Practice
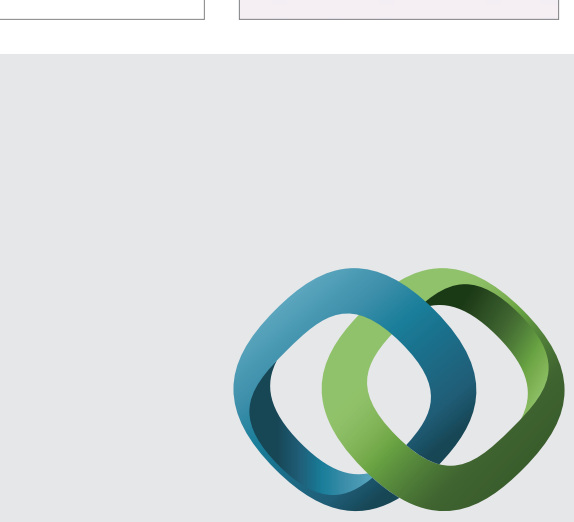

\section{Hindawi}

Submit your manuscripts at

http://www.hindawi.com
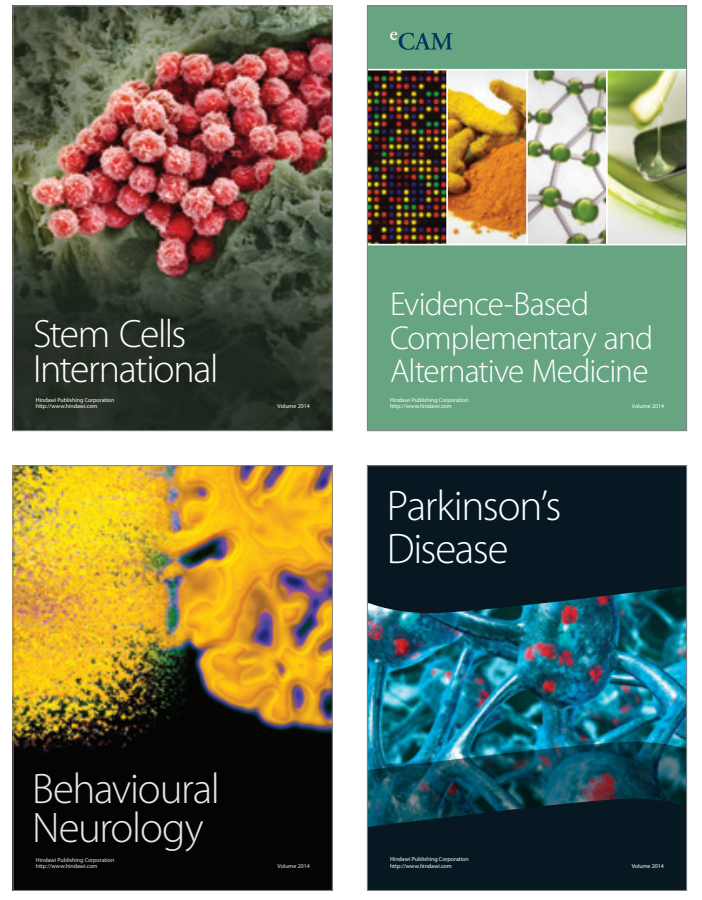
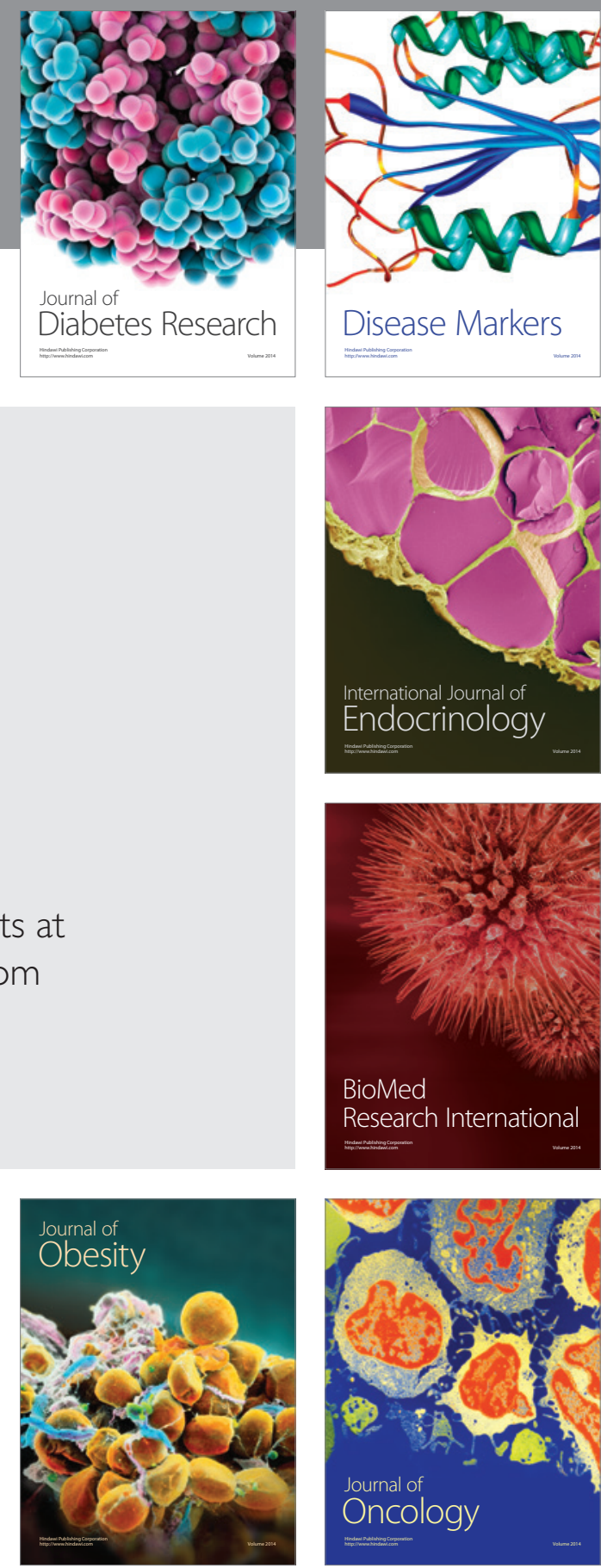

Disease Markers
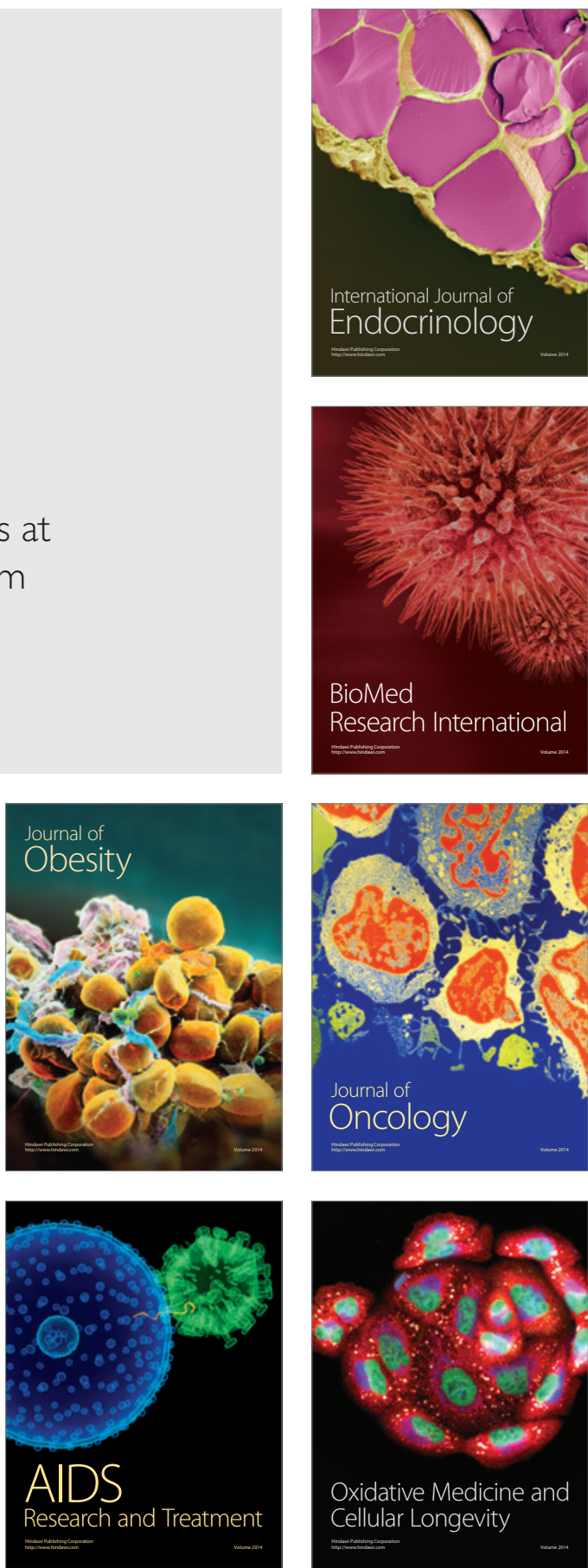